\title{
La mortalité des zones rurales et montagnardes de Catalogne
}

\section{Enric Mendizabal, Anna Mompart, Isabel Pujadas}

\author{
Abstract \\ Mortality in rural and mountainous areas in Catalonia.
}

The authors draw the attention to methodological problems set by the study of mortality in very small and thinly populated unities.

In the example of rural and mountainous areas in Catalonia, migrations disturb extremely the estimate of the accurate mortality levels.

\section{Résumé}

Les auteurs attirent l'attention sur les problèmes méthodologiques posés par l'étude de la mortalité dans des unités territoriales très petites et peu peuplées. Dans l'exemple des zones rurales et de montagne de Catalogne, les migrations perturbent fortement l'appréciation des niveaux exacts de mortalité.

Citer ce document / Cite this document :

Mendizabal Enric, Mompart Anna, Pujadas Isabel. La mortalité des zones rurales et montagnardes de Catalogne. In: Espace, populations, sociétés, 1991-1. Les inégalités géographiques de la mortalité (II) ; The Geographical Inequalities of Mortality (II) pp. 161-164;

doi : 10.3406/espos.1991.1454

http://www.persee.fr/doc/espos_0755-7809_1991_num_9_1_1454

Document généré le 21/03/2016 
Enric MENDIZABAL

Anna MOMPART

Isabel PUJADAS
Centre d'Estudis Demogràfics

Dr. Vilapuig $\mathrm{s} / \mathrm{m}$

Universitat Autonomia de Barcelona

08193 Bellaterra, Barcelona

Espagne

\section{La mortalité des zones rurales et montagnardes de Catalogne}

Notre communication est le résultat d'une recherche sur la population des zones et des comarques de montagne de Catalogne qui a porté sur tous les aspects de la démographie de ce territoire. Elle a pour objectif de présenter les problèmes méthodologi- ques de sources et de données que pose l'étude d'unités territoriales très petites et peu peuplées, comme le sont les municipalités espagnoles et en particulier les zones rurales et montagnardes de Catalogne.

\section{ASPECTS MÉTHODOLOGIQUES DE L'ÉTUDE DE LA MORTALITÉ DES ZONES ET DES COMARQUES DE MONTAGNE}

L'étude spatiale à grande échelle de la mortalité présente des difficultés qui tiennent aux données publiées, à la diversité des structures démographiques et à la différence de taille des populations. Il y a trois difficultés principales à résoudre: en premier lieu, le fait que les données sont publiées selon le lieu d'enregistrement (ce n'est qu'à partir de 1975 qu'elles sont publiées au lieu de résidence); en deuxième lieu, la diversité des structures par âge qui empêche les comparaisons des taux bruts de mortalité ; en troisième lieu, la faiblesse des effectifs entraînant des variations aléatoires.

a) L'enregistrement des statistiques d'étatcivil en Espagne se fait à l'échelon municipal, mais la publication des données de l'Instituto Nacional de Estadistica ne comprenait, jusqu'en 1975, que les provinces et les villes de plus de 20000 habitants. Le principal inconvénient réside dans le fait que les événements étaient enregistrés et publiés par l'I.N.E., selon le lieu d'enregistrement, c'est-à-dire qu'on ne considérait pas le lieu de résidence de la mère dans le cas des naissances, ni la résidence habituelle dans le cas des décès, ni la nouvelle résidence dans le cas des mariages. Depuis 1975, si l'on continue à établir les statistiques d'état-civil au lieu d'enregistrement, elles sont publiées au lieu de résidence. La nouvelle domiciliation résout le problème de surenregistrement des grandes municipalités qui comptent des services hospitaliers et d'assistance sanitaire et où sont les plus fréquemment célébrés les mariages. b) La distribution de la population selon l'âge et le sexe est assez hétérogène parmi les comarques catalanes. Les plus dynami- 
ques se situent autour de Barcelone et tout au long de la côte. Il s'agit de zones urbaines et industrialisées qui ont connu une intense immigration et avaient eu une forte fécondité jusqu'au début des années quatre-vingt, ce qui se manifeste par une structure de population jeune. La Catalogne intérieure et les comarques de monta- gne se caractérisent par une économie essentiellement agraire et un exode rural qui remonte au XIX $\mathrm{X}^{\mathrm{e}}$ siècle; ces régions ont une population vieillie. Les différences maximales se trouvent dans le tableau cijoint, où sont présentées la population la plus jeune: le Baix Llobregat, et la plus âgée: le Priorat.

Tableau 1. Indicateurs de structure par âge

\begin{tabular}{|l|ll|cc|cc|}
\hline & \multicolumn{2}{|c|}{ CATALUNYA } & \multicolumn{2}{c|}{ BAIX LLOBRGAT } & \multicolumn{2}{c|}{ PRIORAT } \\
& 1975 & 1986 & 1975 & 1986 & 1975 & 1986 \\
\hline Population jeune (0-14) & 25,7 & 21,6 & 31,4 & 25,7 & 18,3 & 15,8 \\
Population vieillie (65 +) & 10,2 & 12,3 & 6,3 & 8,3 & 20,4 & 23,6 \\
I. de vieillissement V/J \% & 40 & 57 & 20 & 32 & 111 & 149 \\
Age médian & 30,1 & 33,6 & 26,3 & 30,0 & 43,1 & 40,6 \\
Relation de dépendance \%0 & & & & & & \\
(J V)/A & 55,8 & 51,5 & 60,5 & 51,6 & 63,2 & 65,2 \\
J/A & 39,8 & 32,8 & 50,5 & 39,0 & 29,8 & 26,3 \\
V/A & 16,0 & 18,7 & 10,0 & 12,6 & 33,4 & 39,1 \\
\hline
\end{tabular}

Source: PUJADAS, I.: El envejecimiento de la población: factores demográficos y geográficos y su aplicación en el caso de la población de Catalunya.

Les taux de mortalité détaillés ne sont pas un reflet des niveaux de mortalité réels, mais des différentes structures de la population. Cependant, la standardisation permet de résoudre les différences entre les structures par âge.

Jusqu'en 1985 , on ne dispose pas de données sur la mortalité par âge à l'échelle de la municipalité. C'est la raison pour laquelle il faut faire une standardisation indirecte: aux différentes structures de population comarcales et municipales, on applique les même taux de mortalité : ceux de la Catalogne.

c) Malgré les nombreux regroupements de municipalités dans les zones de montagne, qui se sont produits au cours du siècle, accompagnant le phénomène de dépopulation, il existe encore un nombre important de municipalités à population réduite. La faiblesse de la population entraîne des variations aléatoires, ce qui empêche les études centrées sur une seule année et les études qui se réfèrent à la structure détaillée, eu égard à la faible signification statistique de leur population. Afin de pouvoir donner une certaine fiabilité statistique aux données de population, on analyse la mortalité par groupes de municipalités et à l'échelle de la comarque. Dans certains cas, l'échelle de la comarque cache des situations très différentes, parce qu'elle intègre dans un même ensemble un chef-lieu qui peut être dynamique à de petites municipalités à économie et population en récession. Les groupes étudiés dans ce travail sont: les municipalités de moins de 500 habitants, celles de moins de 2000 habitants, celles qui ont entre 2000 et 5000 habitants, celles de plus de 5000 habitants et l'ensemble de la comarque.

\section{LA MORTALITÉ DANS LES ZONES ET LES COMARQUES DE MONTAGNE EN CATALOGNE}

Le tableau ci-joint indique la mortalité des zones étudiées, après élimination des difficultés méthodologiques par le biais d'une standardisation indirecte qui supprime les différences entre les structures de population, importantes au niveau comarcal, et plus encore au niveau municipal. La Catalogne a un indice standardisé de 1 . Ainsi 
les groupes à indice inférieur à 1 ont un niveau de mortalité plus bas que la moyenne et inversement. Les municipalités ont été regroupées pour obtenir des volumes de population suffisamment importants pour travailler sur des effectifs de même grandeur, partant du postulat qu'à volume de population égal correspondent des caractéristiques socioéconomiques similaires.

Les municipalités dont la population est inférieure à 500 habitants ont toujours des indices standardisés de mortalité inférieurs à ceux des municipalités à population de plus de 5000 habitants. En général plus les communes sont petites, plus leur mortalité est faible. En conséquence on pourrait en déduire, au premier examen, que les municipalités les moins peuplées ont les niveaux de mortalité les plus bas. Les différences territoriales s'expliqueraient par une meilleure qualité de vie dans les zones rurales les moins peuplées, entraînant une moindre mortalité.

Tableau 2. Indice standardisé de la mortalité selon la taille des municipalités. 1987

\begin{tabular}{|l|c|c|c|c|c|}
\hline & -500 & -2000 & $2000-5000$ & +5000 & Comarca \\
\hline Montsec & - & 0,44 & - & - & 0,44 \\
Montseny & 0,93 & 1,08 & 1,01 & 1,08 & 1,03 \\
Guilleries & & & & & \\
Moçanes & 0,79 & 0,93 & - & - & 0,93 \\
Prades & 0,76 & 0,88 & 0,93 & 1,25 & 1,05 \\
Alt Urgell & 0,32 & 0,70 & 0,88 & 1,17 & 0,97 \\
Berguedà & 0,82 & 0,94 & - & 1,25 & 1,06 \\
Cerdenya & 1,10 & 1,06 & 0,98 & 1,14 & 1,09 \\
Garrotxa & 0,48 & 0,60 & 1,06 & 1,84 & 0,89 \\
Pallars Jussà & 0,89 & 1,02 & - & - & 1,02 \\
Pallars Sobirà & 0,99 & 1,06 & 1,10 & 1,27 & 1,14 \\
Ripollès & 0,81 & 0,70 & - & 0,93 & 0,81 \\
Solsonès & 0,95 & 1,08 & 0,95 & - & 1,02 \\
Val d'Aran & -500 & $500-2000$ & $2000-5000$ & +5000 & Total \\
\hline Tous & 0,79 & 0,97 & 1,01 & 1,16 & 1,02 \\
\hline
\end{tabular}

Source: PUJADAS, I.; MENDIZABAL, E.; DEVOLDER, D. ; I CABRE, A. : Estudi demogràfic del territori de muntanya a Catalunya.

Tableau 3. Evolution du mouvement migratoire par comarques et groupes de municipalités. 1976-1981

\begin{tabular}{|c|c|c|c|c|c|c|c|c|c|c|}
\hline \multirow{2}{*}{$\begin{array}{l}\text { Comarques } \\
\text { i zones }\end{array}$} & \multicolumn{2}{|c|}{$>500$} & \multicolumn{2}{|c|}{$500-2000$} & \multicolumn{2}{|c|}{$2000-5000$} & \multicolumn{2}{|c|}{$>5000$} & \multicolumn{2}{|c|}{ Comarca } \\
\hline & tmig & mig & tmig & mig & tmig & mig & tmig & mig & tmig & mig \\
\hline Alt Urgel & -17.34 & $-\quad 271$ & 4.26 & 90 & 4.40 & -47 & 8.90 & 458 & 2.32 & 230 \\
\hline Berguèda & -47.80 & -971 & -28.27 & -1477 & -5.81 & -125 & 3.46 & 434 & -9.75 & -2140 \\
\hline Cerdanya & -21.11 & -245 & -10.87 & -233 & - & - & -15.83 & -487 & -15.13 & -966 \\
\hline Garrotxa & -31.76 & $-\quad 394$ & -6.37 & 345 & -15.05 & -615 & 9.74 & 1231 & $-\quad 0.54$ & -124 \\
\hline P. Jussà & $-\quad 3.40$ & $-\quad 43$ & -18.68 & -445 & 14.98 & 474 & 5.51 & -162 & 1.80 & 176 \\
\hline P. Sobirà & -27.07 & -501 & 4.60 & 49 & - & - & - & - & 15.37 & 451 \\
\hline Ripollès & -27.71 & -553 & 2.68 & 30 & -4.26 & -335 & 5.04 & 313 & $-\quad 3.53$ & 605 \\
\hline Solsonès & -31.78 & -638 & 7.35 & 34 & - & - & 1.02 & 30 & -12.40 & 703 \\
\hline Val d'Aran & -0.19 & $-\quad 1$ & 0.52 & 6 & 34.71 & 473 & - & - & 16.61 & 478 \\
\hline Montsec & - & - & -43.09 & $-\quad 301$ & - & - & - & - & -43.09 & 301 \\
\hline Montseny & -18.09 & -506 & 2.36 & 116 & 1.79 & 212 & 1.59 & 95 & $-\quad 0.44$ & - \\
\hline Prades-M. & -17.61 & -68 & 2.08 & $-\quad 28$ & - & - & - & - & 5.48 & $\begin{array}{l}-\quad 97\end{array}$ \\
\hline Tous & -25.31 & -4193 & 9.42 & -2632 & 0.08 & 36 & 3.58 & 1851 & 3.92 & -4938 \\
\hline
\end{tabular}

Source: PUJADAS, I.; MENDIZABAL, E.; DEVOLDER, D.; I CABRE, A. : Estudi demogràfic del territori de muntanya a Catalunya. 
Tableau 4. Soldes migratoires par âge. 1975-1981 (en pour mille)

\begin{tabular}{|c|c|c|c|c|c|c|c|c|c|c|}
\hline Edat & \multicolumn{2}{|c|}{$\begin{array}{l}\text { Mun. }<500 \\
\text { tot. } \quad \text { prob. }\end{array}$} & \multicolumn{2}{|c|}{$\begin{array}{c}\text { Mun. } 500 \text { à } 2000 \\
\text { tot. } \quad \text { prob. }\end{array}$} & \multicolumn{2}{|c|}{$\begin{array}{l}\text { Mun. } 2000 \text { à } 5000 \\
\text { tot. } \quad \text { prob. }\end{array}$} & \multicolumn{2}{|c|}{$\begin{array}{ll}\text { Mun. } & <5000 \\
\text { tot. } & \text { prob. }\end{array}$} & \multicolumn{2}{|c|}{$\begin{array}{l}\text { Chef-lieu } \\
\text { tot. prob. }\end{array}$} \\
\hline $0-4$ & 47 & 5.76 & 125 & 7.61 & $-\quad 32$ & -1.39 & -74 & 2.00 & 67 & 0.79 \\
\hline $5-9$ & -12 & $-\quad 1.27$ & -186 & -10.00 & 90 & 4.18 & -252 & 6.88 & 363 & 4.21 \\
\hline $10-14$ & -326 & -25.18 & -282 & -13.06 & 194 & 8.32 & -368 & 9.88 & 46 & 0.48 \\
\hline $15-19$ & -482 & -34.78 & -499 & -21.02 & 33 & 1.36 & 54 & 1.35 & 896 & -8.80 \\
\hline $20-24$ & -674 & -51.20 & -511 & -24.36 & 17 & -0.78 & 111 & 3.23 & -1092 & -12.08 \\
\hline $25-29$ & -413 & -38.39 & -336 & -19.12 & 79 & -3.88 & 7 & 0.21 & -822 & $-\quad 9.97$ \\
\hline $30-34$ & -43 & $-\quad 5.04$ & -165 & -10.94 & 52 & 3.00 & 30 & 1.05 & 185 & 2.66 \\
\hline $35-39$ & -97 & -1191 & -190 & -13.71 & 20 & 1.26 & 84 & 3.09 & 352 & 5.41 \\
\hline $40-44$ & -188 & $-11,34$ & -254 & -12.51 & 145 & 6.94 & $-\quad 1$ & 0.03 & 241 & 2.74 \\
\hline $45-49$ & -188 & -13.84 & -309 & -13.58 & -121 & -5.42 & 104 & 2.84 & 516 & 5.41 \\
\hline $50-54$ & -346 & -24.12 & -307 & -13.52 & -201 & -8.89 & -94 & $-\quad 2.54$ & 953 & 9.83 \\
\hline $55-59$ & -271 & -22.17 & -215 & -12.17 & -45 & -2.68 & 351 & 13.08 & 180 & 2.45 \\
\hline $60-64$ & -169 & -14.77 & -27 & $-\quad 1.71$ & -116 & -7.36 & 341 & 13.72 & 28 & 0.41 \\
\hline $65-69$ & -146 & -14.00 & -68 & $-\quad 4.49$ & -7 & -0.52 & 101 & 4.36 & 120 & 1.92 \\
\hline
\end{tabular}

Source: PUJADAS, I. ; MENDIZABAL, E. ; DEVOLDER, D. ; I CABRE, A. : Estudi demogràfic del territori de muntanya a Catalnya.

Mais l'explication doit venir des migrations qui ont affecté les structures démographiques des zones de montagne de façon intense. Ainsi les ménages composés d'isolés âgés sont très nombreux, or dès qu'elles sont affectées par des problèmes d'assistance ou de santé, ces personnes se déplacent vers les centres urbains où se trouvent leurs familles.

L'analyse des migrations selon la taille de la municipalité et selon l'âge explique la distribution de l'indice standardisé de mortalité. Entre 1976 et 1981, dans les unités de moins de 500 habitants, le solde migratoire est toujours négatif, tandis que dans les unités de plus de 5000 habitants, le sens des migrations s'inverse et le solde est positif dans 5 des 8 comarques qui atteignent ce chiffre.

La probalité de migrer selon l'âge et selon la taille de la population est supérieure pour les personnes âgées des petites unités qui partent vivre dans les municipalités plus importantes. Aussi le risque de mortalité touche-t-il davantage les personnes âgées qui migrent vers de plus grandes villes pour rejoindre leur famille, et les grands malades qui sont envoyés dans les centres hospitaliers importants de Barcelone ou des autres villes.

On ne peut parvenir à une appréciation exacte des niveaux de mortalité dans les zones de montagne, car il y a sousestimation de la mortalité réelle, liée, ainsi que cela a été souligné, à l'émigration différentielle selon l'âge et l'état de santé.

\section{BIBLIOGRAPHIE}

PUJADAS 1.: «El envejecimiento de la poblacion: factores demográficos y geográficos y su applicación en el caso de la población de Catalunya » a Envejecimiento y sociedad (siglos $X X$ y $X X I$ ), a l'Hospitalet de Llobregat, 16 y 17 de maig de 1988.

PUJADAS I., MENDIZABAL E. : «La poblacio de muntanya a Catalunya", a Treballs de la Societat Catalana de Geografia, $\mathrm{n}^{\circ} 12$, Barcelona, stembre 1987, pp. 93-111.

PUJADAS I., MENDIZABAL E., CABRE A. : «La poblacio de la muntanya gironina" a Revista de Girona, $\mathrm{n}^{\circ} 119$, novembre-desembre 1986, Girona, pp. 29-34.

PUJADAS I., MENDIZABAL E., DEVOLDER D., CABRE A. : «Estudi demogràfic del territori de muntanya a Catalunya », a Papers de Demografia, $n^{\circ} 18$, 1987, Bellaterra (Barcelona), $139 \mathrm{p}$.
PUJADAS I., CABRE A., DEVOLDER D., MENDIZABAL E., MASSO J., VAQUE E. : dotze informes publicats per al Servei d'Accio Comarcal, Direccio Genreal de Politica Territorial, Barcelona, 1987. Estudi demogràfic de la comarca de l'Alt Urgell, 222 p.

Id. del Berguedà, 232 p.

Id. de la Cerdanya, $193 \mathrm{p}$.

Id. de la Garrotxa, 243 p.

Id. del Pallars Jussà, 237 p.

Id. del Pallars Sobirà, 199 p.

Id. del Ripollès, 243 p.

Id. del Solsonès, $174 \mathrm{p}$.

Id. de la Vall d'Aran, $158 \mathrm{p}$.

Estudi demogràfic de la zona del Montsec, $111 \mathrm{p}$.

Id. de Prades-Montsant, $131 \mathrm{p}$.

Id. del Montseny-Lluçanès-Guilleries, $240 \mathrm{p}$. 